\title{
Unusual Cause of Acute Sinusitis and Orbital Abscess in COVID-19 Positive Patient
}

\author{
Courtney Shires ${ }^{1}$ and Theodore Klug $^{1}$ \\ ${ }^{1}$ West Cancer Center
}

August 23, 2020

\begin{abstract} spontaneously drained this bacteria through the skin, an uncommon occurrence with orbital abscesses.

Unusual Cause of Acute Sinusitis and Orbital Abscess in COVID-19 Positive Patient

Courtney B. Shires, MD, FACS ${ }^{1}$; Theodore Klug, MD, MPH ${ }^{1}$

${ }^{1}$ West Cancer Center, 7945 Wolf River Blvd, Germantown, TN 38104

Corresponding Author:

Theodore Klug, MD, MPH

West Cancer Center

7945 Wolf River Blvd, Germantown, TN 38104

901-4911721

theodklu@gmail.com

Author Contributions

Courtney B. Shires, MD, FACS: Collected data, wrote and edited article

Theodore Klug, MD, MPH: Collected data, wrote and edited article
\end{abstract}

Peptoniphilus indolicus is not usually seen in the eye but is a commensal of the human vagina and gut. However, with COVID-19, eye infections and other unusual complications are possible with unsuspected bacteria. The patient presented here

\section{Key Words}

COVID-19, Peptoniphilus indolicus, sinusitis, orbital abscess

Financial Support: This particular research received no internal or external grant funding.

Key clinical message : COVID-19 can affect the presence of bacteria within certain anatomical regions. Specifically, Peptoniphilus indolicus is not normally found outside of the vagina or gut biome, but was found in the sinus and orbit of our patient.

\section{Abstract}

\section{Background}

Peptoniphilus indolicus is not usually seen in the eye but is a commensal of the human vagina and gut. However, with COVID-19, eye infections and other unusual complications are possible with such unsuspected 
bacteria. The patient presented here spontaneously drained this bacteria through the skin, an uncommon occurrence with orbital abscesses.

\section{Case Presentation}

The patient is a 76-year-old white male from a nursing home tested positive for COVID-19 and was sent from a nursing facility for left eye drainage and psychiatric evaluation. Upon presentation, the patient was not fully oriented and could not provide a history of the eye drainage. CT scan showed sinusitis with left orbital and periorbital abscess formation, cellulitis, and extensive osteomyelitis. He underwent endoscopic transnasal drainage and orbiotomy. Cultures returned positive for MRSA, Streptococcus constellatus, and Peptoniphilus indolicus. He was maintained on several days of IV antibiotics and returned to the nursing home.

\section{Conclusions}

Given the concomitant infection with COVID-19 and unusual presentation, the patient's sinus cultures support the notion that COVID-19 can affect the presence of bacteria within certain anatomical regions. Specifically, Peptoniphilus indolicus is not normally found outside of the vagina or gut biome. Avascular, pale mucosa and bone of the nasal cavity was noted during surgery of this COVID-19 infected patient, which is in contrast to the friable and edematous tissue typically found in acutely infected sinuses. Our patient's orbital abscess began to drain spontaneously through the skin, which is rare for orbital abscesses.

\section{Background}

Peptoniphilus indolicus is not usually seen in the eye but is a commensal of the human vagina and gut. However, with COVID-19, eye infections and other unusual complications are possible with such unsuspected bacteria. The patient presented here spontaneously drained through the skin, an uncommon occurrence with orbital abscesses.

\section{Case Presentation}

The patient is a 76-year-old white male who tested positive for COVID-19 but was sent from a nursing facility for left eye drainage and "psychiatric evaluation." Upon presentation, the patient was not fully oriented and could not provide a history of the eye drainage. His past medical history is significant for hypertension, Type 2 diabetes mellitus requiring insulin, diabetic ketoacidosis, testicular cancer, and transient ischemic attack. CT scan upon presentation showed sinusitis with left orbital and periorbital abscess formation, cellulitis, and extensive osteomyelitis (Figures 1-3). The margin of the orbital abscess was within the inferior margin of the globe, where there appeared to be an open defect within the globe itself. The patient also had a chest x-ray showing some patchy infiltrates, particularly in the right lung, consistent with a COVID-19 infection.

The patient was admitted and subsequently underwent endoscopic left middle turbinate reduction, endoscopic left maxillary antrostomy with tissue removal, and endoscopic anterior ethmoidectomy with the Otolaryngology service the following day. The patient also underwent left orbiotomy with drainage of the left orbital abscess with the Opthalmology service that same day. During surgery, his nasal cavity was oddly avascular (Figure 4). The turbinate was edematous but pale. The mucosa did not bleed when manipulated. When the bone of the middle turbinate and medial wall of the maxilla were removed with Tru cut forceps, they did not bleed. The fat of the orbit was pale. Cultures were taken during surgery and returned positive for MRSA, Streptococcus constellatus, and Peptoniphilus indolicus. He completed several days of intravenous antibiotics during the hospitalization but continued to have drainage from his left eye (Figures 5-6). Otolaryngology evaluated him daily and had no plans to do further surgery. He was deemed hemodynamically and neurologically stable and was transferred back to his nursing facility for completion of intravenous antibiotics.

\section{Discussion}

Rare disease processes and manifestations have been reported in COVID-19 positive patients. Peptoniphilus indolicus, a type of bacteria normally found in the vagina and stomach $^{1}$, was found within our patient's 
orbit. There are now more than 15 Peptoniphilus species within the genus, seven of which were discovered in $2012^{2-9}$. Still, up until now, none of the 15 species have been seen within the orbit. To date, Peptoniphilus species have most commonly been associated with diabetic skin and soft tissue infections, bone and joint infections, and surgical site infections ${ }^{10-13}$. A recent study of pre-term labor and early neonatal sepsis also isolated Peptoniphilus from amniotic fluid causing choramnionitis ${ }^{14}$. Only recently did a case series of Peptoniphilus causing bloodstream infection (BSI), either alone or as part of a polymicrobial infection, become available ${ }^{15}$. We believe that our case is the first to document Peptoniphilus within the orbit. Physicians should therefore add it to their differential when COVID-19 patients present with sinusitis and orbital abscess. An Infectious Disease team should also be a part of the care team in order to adequately cover unusual organisms.

Of note, the avascularity of the nasal tissue was of significant interest and very peculiar to the surgeons involved with the case. Limb ischemia and avascular surgical fields have been noted during the COVID19 pandemic, as evidenced in the Italian Lombardy region ${ }^{16}$. In Lombardy, the incidence of acute limb ischemia (ALI ) significantly increased during the COVID-19 pandemic and successful revascularization was lower than expected, believed to be due to a virus-related hypercoagulable state ${ }^{16}$. As reported by Silingardi and colleagues, the increased thromboembolic complications in COVID-19 patients have been reported even in those receiving anticoagulant therapy and in nonatherosclerotic patients ${ }^{16-19}$. In turn, precautions must be taken when performing surgery on COVID-19 positive patients, specifically from a vascular standpoint.

Finally, the Ophthalmology service made note of the peculiarity of the spontaneously-draining orbital abscess. Typical orbital abscess presentations include red eye, proptosis, ophthalmoplegia, and pain ${ }^{20-21}$. In severe cases, the optic nerve can become compressed, leading to compressive optic neuropathy ${ }^{21}$. The incidence of abscess formation among sinus disease patients varies from $6.25 \%$ to $20 \%$, to as high as $78.6 \%{ }^{22-25}$. Rarely, however, does an orbital abscess drain on its own. According to the American Academy of Ophthalmology, current guidelines recommend surgical drainage in conjunction with intravenous antibiotics to achieve complete resolution of the infection in patients over the age of fourteen ${ }^{26}$. Still, there are very few studies with high power looking at orbital abscess drainage in adults. For example, from a study by Kayhan and colleagues with ten total patients, external drainage of the abscess was needed in six of the patients in the study ${ }^{27}$. Nevertheless, zero of the six orbital abscesses that needed draining did so spontaneously, further supporting the rarity of the type of orbital abscess found in the COVID-19 positive patient in our study.

\section{Conclusions}

Given the concomitant infection with COVID-19 and unusual presentation, the patient's cultures support the notion that COVID-19 can affect the presence of bacteria within certain anatomical regions. Specifically, Peptoniphilus indolicus is not normally found outside of the vagina or gut biome. Like other parts of the body, the sinonasal cavity can become avascular as a result of COVID infections. It is also rare for orbital abscesses to drain spontaneously, as this abscess did.

\section{List of Abbreviations}

Methicillin resistant staph aureus (MRSA), Coronavirus Disease 2019 (COVID-19), Computed tomography (CT), diabetes mellitus (DM), bloodstream infection (BSI), acute limb ischemia (ALI)

Conflicts of Interest: The authors report no relevant financial disclosures related to this current work.

Ethical Considerations: All issues related to ethics were taken into consideration throughout the study design and proposal and implemented during the research study itself.

Consent : Informed consent was obtained by the study patient, beneficence was made a top priority, and respect for confidentiality and privacy were upheld during the study and its various analysis and information assertation components.

\section{References}


1. Ezaki T, Kawamura Y, Li N, Li ZY, Zhao L, Shu S. Proposal of the genera Anaerococcus gen. nov., Peptoniphilus gen. nov. and Gallicola gen. nov. for members of the genus Peptostreptococcus. Int $J$ Syst Evol Microbiol . 2001;51(Pt 4):1521-1528. doi:10.1099/00207713-51-4-1521

2. Cho E, Park SN, Kim HK, et al. Draft genome sequence of the novel Peptoniphilus sp . strain ChDC B134, isolated from a human periapical abscess lesion. Genome Announc. 2013;1(pii):e00822-13

3. Citron DM, Tyrrell KL, Goldstein EJ. Peptoniphilus coxii sp . nov. and Peptoniphilus tyrrelliae sp . nov. isolated from human clinical infections. Anaerobe. 2012;18:244-248.

4. Kim DS, Jung MY, Kang A, et al. Genome sequence of Peptoniphilus rhinitidis 1-13T, an anaerobic coccus strain isolated from clinical specimens. J Bacteriol. 2012;194:2405-2406.

5. Mishra AK, Hugon P, Lagier JC, et al. Non contiguous-finished genome sequence and description of Peptoniphilus obesi sp . nov. Stand Genomic Sci. 2013;7:357-369.

6. Mishra AK, Hugon P, Robert C, Raoult D, Fournier PE. Non contiguous-finished genome sequence and description of Peptoniphilus grossensis sp . nov. Stand Genomic Sci. 2012;7:320-330.

7. Rooney AP, Swezey JL, Pukall R, Schumann P, Spring S. Peptoniphilus methioninivorax sp . nov., a Gram-positive anaerobic coccus isolated from retail ground beef. Int J Syst Evol Microbiol. 2011;61:1962-1967.

8. Song Y, Liu C, Finegold SM. Peptoniphilus gorbachii sp . nov., Peptoniphilus olsenii sp . nov., and Anaerococcus murdochii sp . nov. isolated from clinical specimens of human origin. J Clin Microbiol. 2007;45:1746-1752.

9. Ulger-Toprak N, Lawson PA, Summanen P, O'Neal L, Finegold SM. Peptoniphilus duerdenii sp . nov. and Peptoniphilus koenoeneniae sp . nov., isolated from human clinical specimens. Int J Syst Evol Microbiol. 2012;62:2336-2341.

10. Dowd SE, Wolcott RD, Sun Y, McKeehan T, Smith E, Rhoads D. Polymicrobial nature of chronic diabetic foot ulcer biofilm infections determined using bacterial tag encoded FLX amplicon pyrosequencing (bTEFAP) PLoS One. 2008;3:e3326.

11. Wolcott RD, Gontcharova V, Sun Y, Dowd SE. Evaluation of the bacterial diversity among and within individual venous leg ulcers using bacterial tag-encoded FLX and titanium amplicon pyrosequencing and metagenomic approaches. BMC Microbiol. 2009;9:226.

12. Smith DM, Snow DE, Rees E, et al. Evaluation of the bacterial diversity of pressure ulcers using bTEFAP pyrosequencing. BMC Med Genomics. 2010;3:41.

13. Walter G, Vernier M, Pinelli PO, et al. Bone and joint infections due to anaerobic bacteria: an analysis of 61 cases and review of the literature. Eur J Clin Microbiol Infect Dis. 2014 ; [Epub ahead of print]

14. Wang X, Buhimschi CS, Temoin S, Bhandari V, Han YW, Buhimschi IA. Comparative microbial analysis of paired amniotic fluid and cord blood from pregnancies complicated by preterm birth and early-onset neonatal sepsis. PLoS One. 2013;8:e56131.

15. Brown K, Church D, Lynch T, Gregson D. Bloodstream infections due to Peptoniphilus spp.: report of 15 cases. Clin Microbiol Infect . 2014;20(11):O857-O860. doi:10.1111/1469-0691.12657

16. Bellosta R, Luzzani L, Natalini G, et al. Acute limb ischemia in patients with COVID-19 pneumonia [published online ahead of print, 2020 Apr 29]. J Vasc Surg . 2020;S0741-5214(20)31080-6. doi:10.1016/j.jvs.2020.04.483

17. Silingardi R, Gennai S, Migliari M, Covic T, Leone N. Acute limb ischemia in COVID-19 patients: Could aortic floating thrombus be the source of embolic complications? [published online ahead of print, 2020 Jun 17]. J Vasc Surg . 2020;S0741-5214(20)31351-3. doi:10.1016/j.jvs.2020.06.008

18. Perini P., Nabulsi B., Massoni C.B., Azzarone M., Freyrie A. Acute limb ischaemia in two young, non-atherosclerotic patients with COVID-19. Lancet. 2020;395:1546.

19. 3. Helms J., Tacquard C., Severac F., Leonard-Lorant I., Ohana M., Delabranche X. CRICS TRIGGERSEP Group (Clinical Research in Intensive Care and Sepsis Trial Group for Global Evaluation and Research in Sepsis). High risk of thrombosis in patients with severe SARS-CoV-2 infection: a multicenter prospective cohort study. Intensive Care Med. 2020;46:1089-1098.

20. Chandler JR, Langenbrunner DJ, Steven ER. The pathogenesis of orbital complications in acute sinusitis. Laryngoscope. 1970;80:1414-28. 
21. Lam Choi VB, Yuen HK, Biswas J, Yanoff M. Update in pathological diagnosis of orbital infections and inflammations. Middle East Afr J Ophthalmol . 2011;18(4):268-276. doi:10.4103/0974-9233.90127

22. Morgan PR, Morrison WV. Complications of frontal and ethmoid sinusitis. Laryngoscope . 1980;90(4):661-666. doi:10.1288/00005537-198004000-00013

23. Welsh LW, Welsh JJ. Orbital complications of sinus diseases. Laryngoscope . 1974;84(5):848-856. doi:10.1288/00005537-197405000-00015

24. Giletto JB, Scherr SA, Mikaelian DO. Orbital complications of acute sinusitis in children. Trans Pa Acad Ophthalmol Otolaryngol . 1981;34(1):60-64.

25. Chaudhry IA, Al-Rashed W, Arat YO. The hot orbit: orbital cellulitis. Middle East Afr J Ophthalmol . 2012;19(1):34-42. doi:10.4103/0974-9233.92114.

26. Ophthalmology 2000;107:1454-1458

27. Kayhan FT, Sayin I, Yazici ZM, Erdur O. Management of orbital subperiosteal abscess. J Craniofac Surg . 2010;21(4):1114-1117. doi:10.1097/SCS.0b013e3181e1b50d

\section{Figure Legend :}

Figure 1. Axial sinus CT.

Figure 2. Coronal Sinus CT.

Figure 3. Sagittal Sinus CT.

Figure 4. Endoscopic view of avascular tissue of the left middle meatus. Diamond- left middle turbinate, Star- left maxillary os. Figure 5. Orbital abscess drainage - eye open. Figure 6. Orbital abscess drainage eye closed.

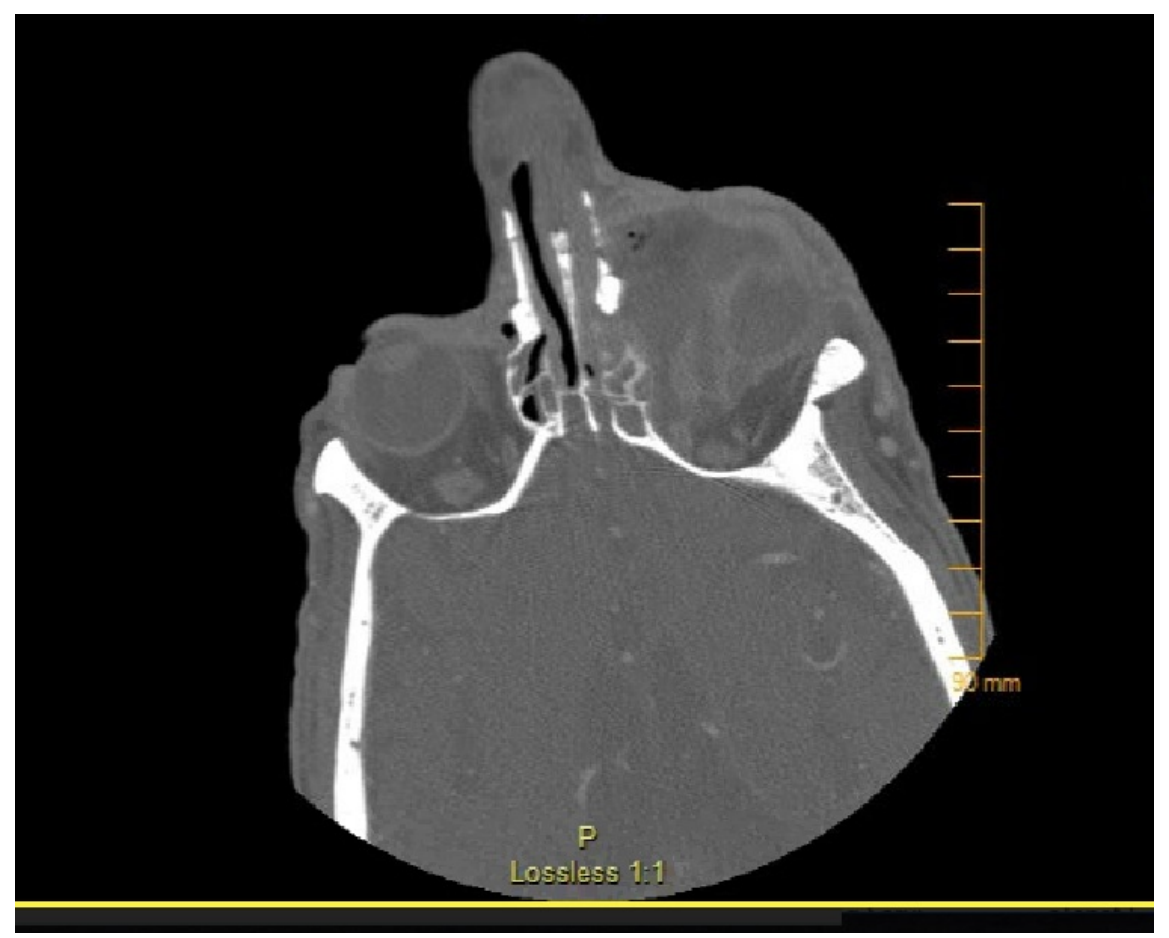

Figure 1. Axial sinus CT. 


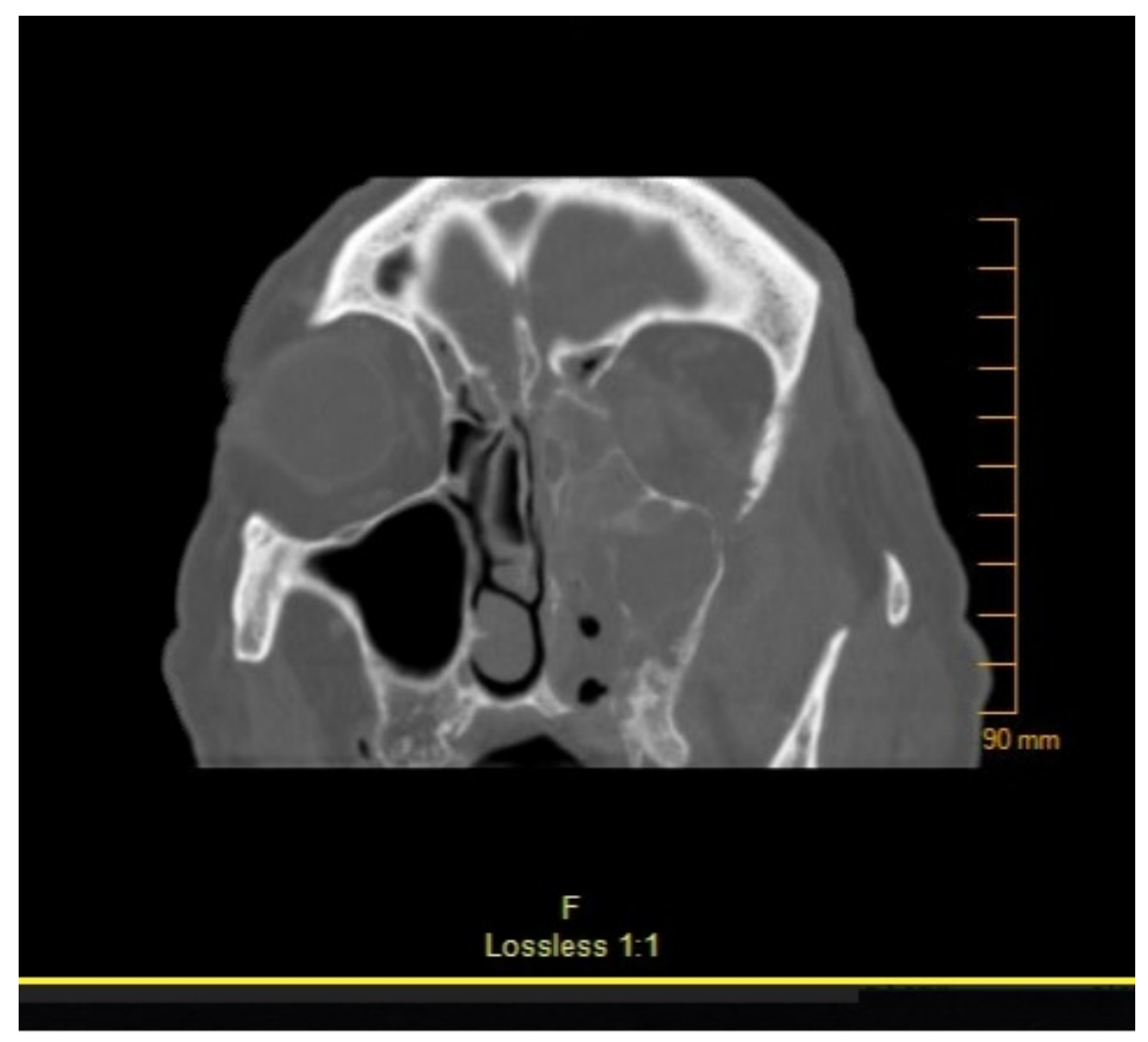

Figure 2. Coronal Sinus CT.

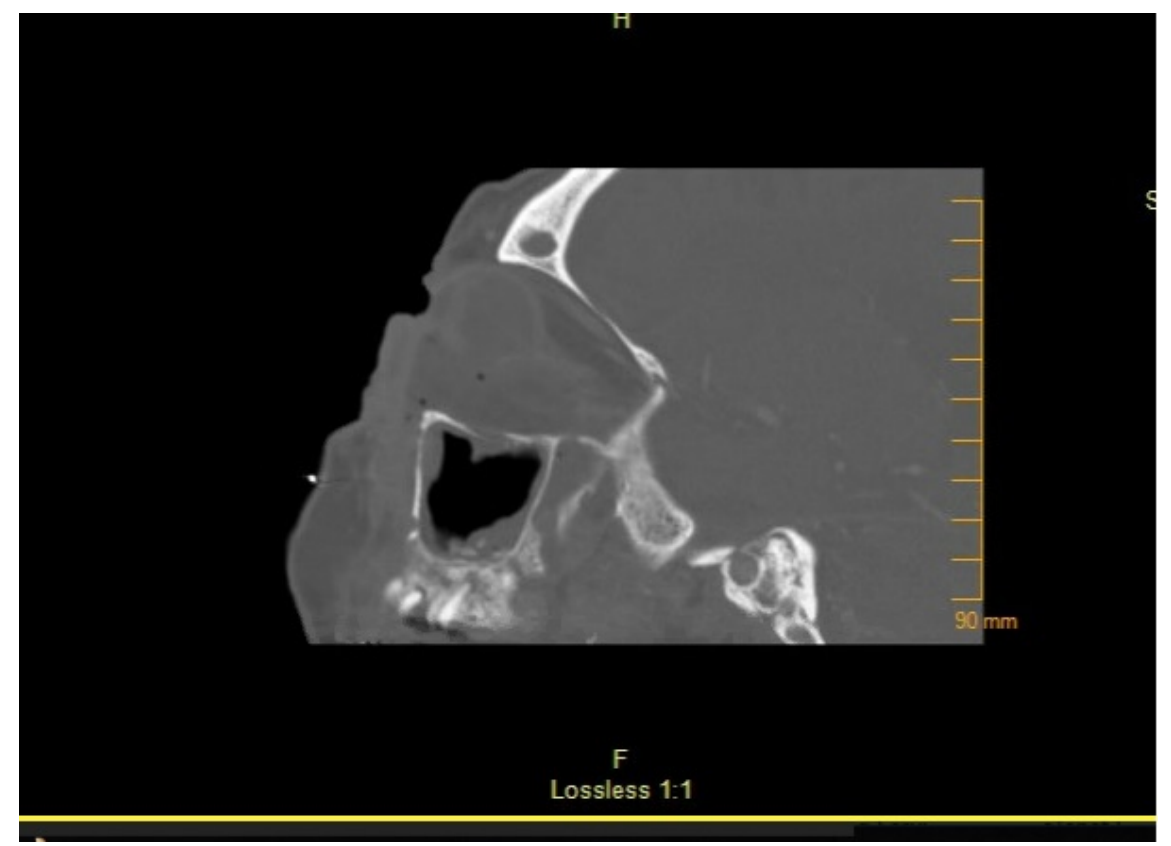

Figure 3. Sagittal Sinus CT. 


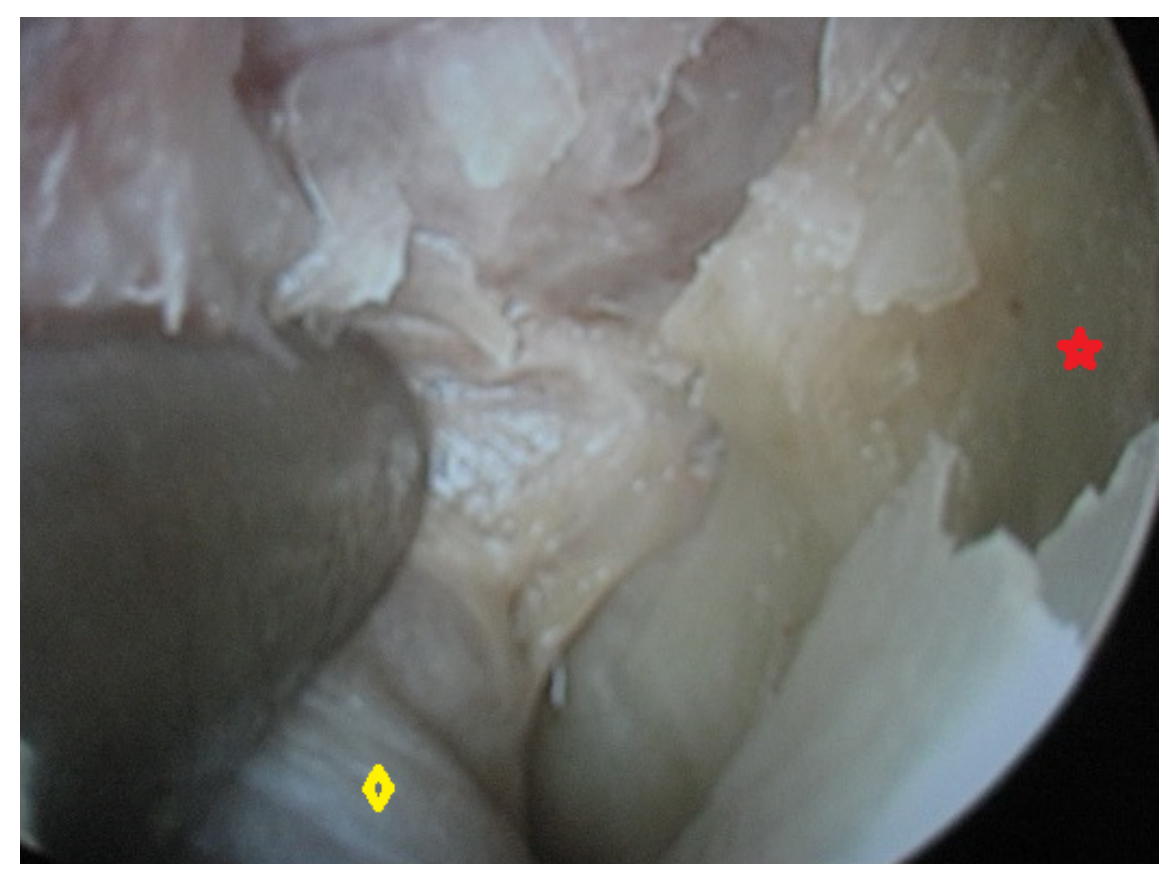

Figure 4. Endoscopic view of avascular tissue of the left middle meatus. Diamond- left middle turbinate, Star- left maxillary os.

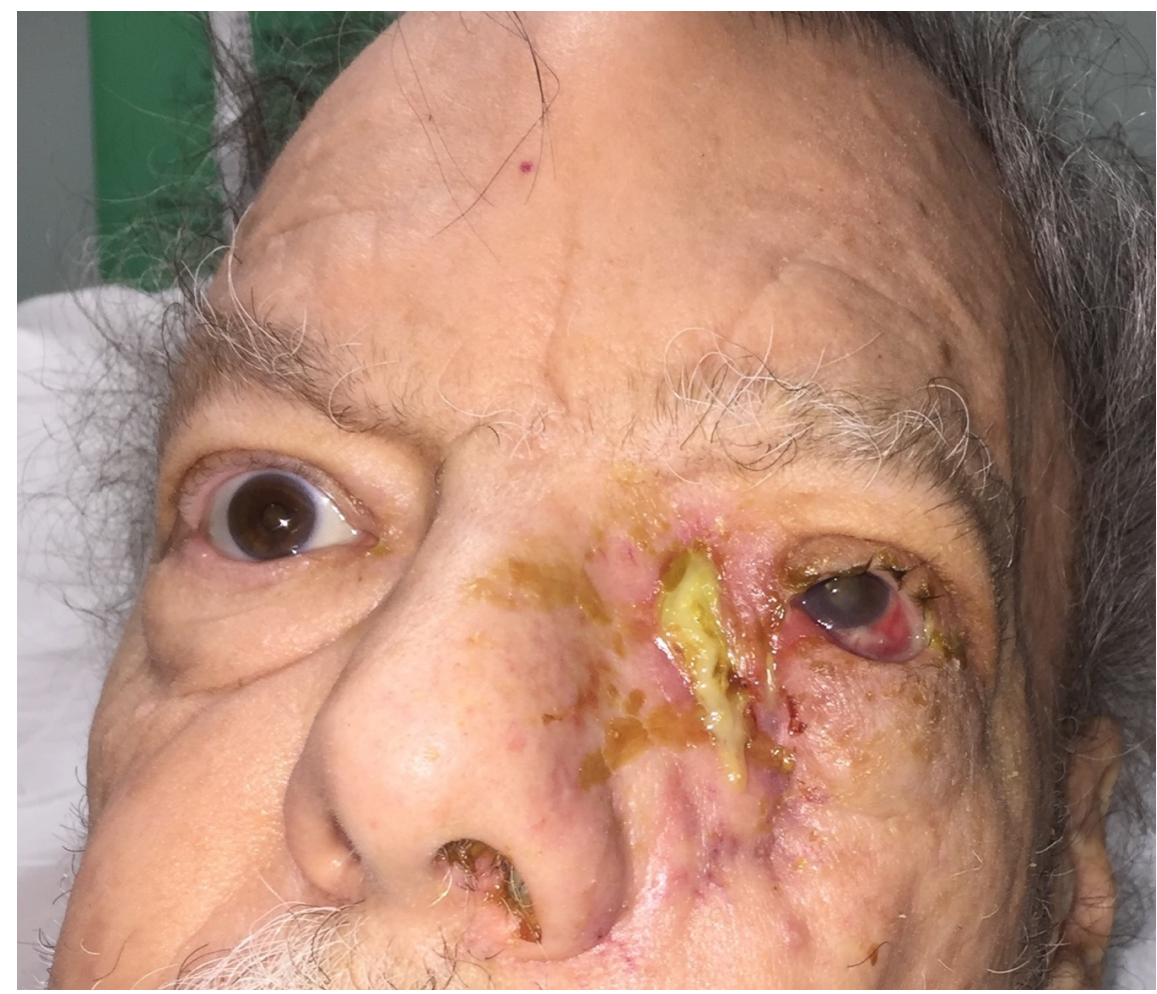

Figure 5. Orbital abscess drainage - eye open. 


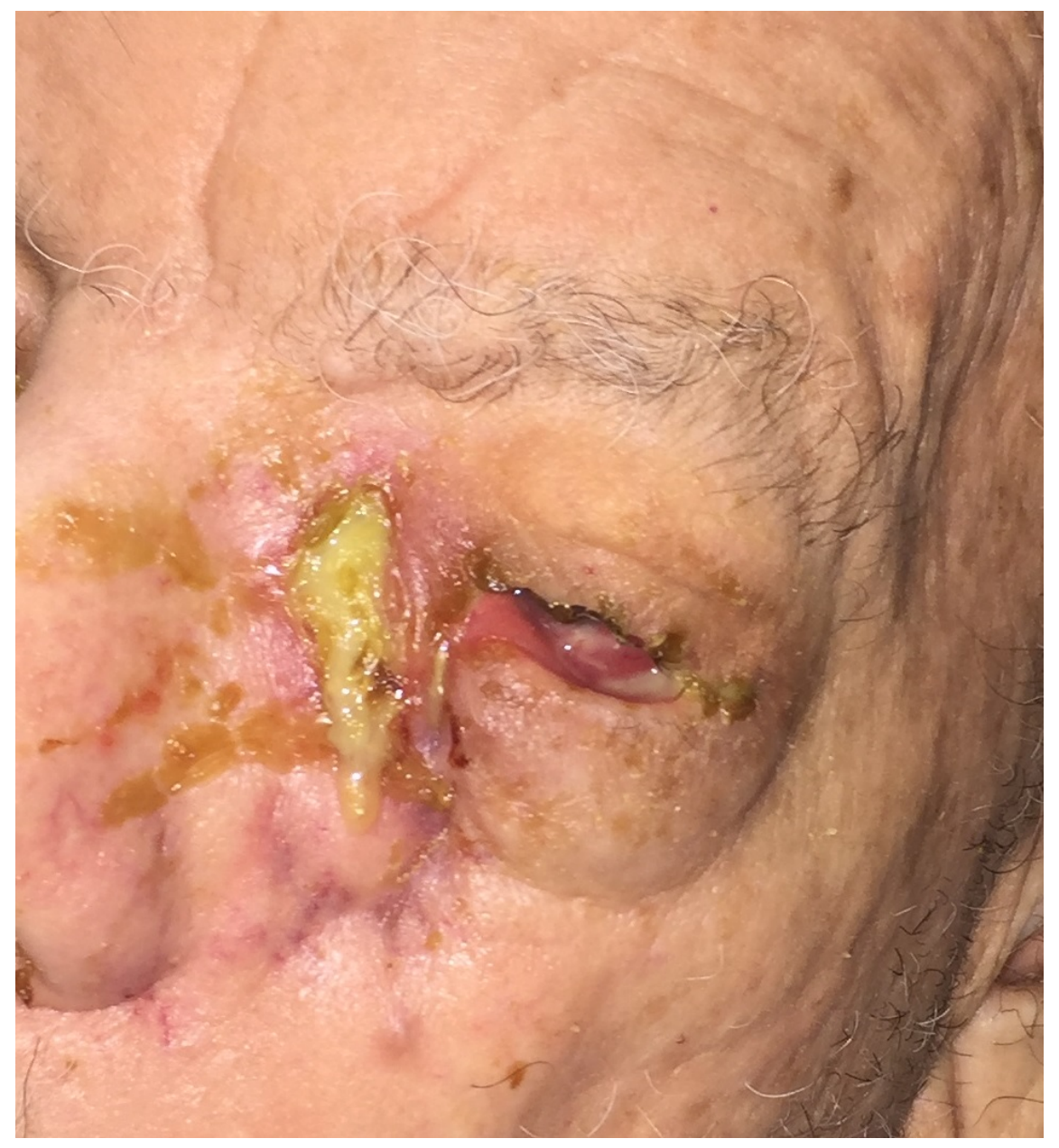

Figure 6. Orbital abscess drainage - eye closed. 